Applied Optics (Lasers, Photonics, and Environmental Optics) 39 (21) 3738-3745

\title{
Simultaneous Light Scattering and Intrinsic Fluorescence Measurement for the Classification of Airborne Particles
}

\author{
Paul H. Kaye, John E. Barton, Edwin Hirst \\ Science and Technology Research Centre, University of Hertfordshire, Hatfield, Hertfordshire, AL10 \\ 9AB, U.K. \\ and James M. Clark \\ DERA CBD, Porton Down, Salisbury, Wiltshire SP4 0JQ, U.K.
}

\begin{abstract}
This paper describes a prototype laboratory light scattering instrument which integrates two approaches to airborne particle characterisation: spatial light scattering analysis and intrinsic fluorescence measurement, with the aim of providing an effective means of classifying biological particles within an ambient aerosol. The system uses a single continuous-wave $266 \mathrm{~nm}$ ultraviolet laser to generate both the spatial elastic scatter data (from which an assessment of particle size and shape is made), and the particle intrinsic fluorescence data, from particles in the approximate size range 1$10 \mu \mathrm{m}$ diameter carried in a sample airflow through the laser beam. Preliminary results suggest that this multi-parameter measurement approach can provide an effective means of classifying different particle types and can reduce occurrences 'false positive' detection of biological aerosols.
\end{abstract}

\section{OCIS CODES:}

$\begin{array}{lllll}120.5820 & 120.1880 & 290.5820 & 290.1090 & 300.2530\end{array}$ 


\section{INTRODUCTION}

Driven by environmental, occupational, and defense concerns, there has been growing interest in methods which offer the potential of analyzing and characterizing airborne particles in real-time. A particular objective has been to achieve differentiation between biological and non-biological particles and (ideally) between various types of biological particles such that potential pathogens may be detected. Light scattering based instruments such as optical particle counters (OPCs) or aerodynamic particle sizers have long been a favored choice for airborne particle count and size monitoring as they offer close to real-time response and can operate continuously without the need for reagents, etc. For the discrimination of biological particles, particle intrinsic fluorescence offers significant potential and several workers have developed systems which incorporate particle fluorescence measurement in conjunction with the measurement of other particle parameters in an attempt to optimize discrimination.

In 1995, Pinnick et al ${ }^{1}$ described a laser-based particle counter that detected fluorescence and elastic scattering from individual airborne particles as they traversed the beam from a 488nm argon-ion laser. The low levels of intrinsic fluorescence observed from kaolin, hematite and polystyrene particles in comparison to that observed from several types of biological particles suggested that the instrument would be useful in discriminating biological from non-biological particles. In an effort to enhance particle discrimination, the same group went on to extend the capabilities of the system to record the

spectrum of fluorescence from a particle rather than simply the fluorescence magnitude ${ }^{2,3}$. More recently (1999) this work has been further developed by the use of one of two ultra violet excitation frequencies in place of the $488 \mathrm{~nm}$ beam. Results ${ }^{4}$ showing the fluorescence spectra from individual 2$5 \mu \mathrm{m}$ biological aerosol particles excited by either $266 \mathrm{~nm}$ or $351 \mathrm{~nm}$ radiation from a Q-switched laser (Nd-YAG and Nd-YLF respectively) illustrate the differences in fluorescence spectra which can be observed from different biological particles. 
In 1997, Hairston et $a l^{5}$ described an instrument for the real-time detection of bioaerosols which incorporated simultaneous measurement of particle aerodynamic size and intrinsic fluorescence. The measurement of aerodynamic size in conjunction with fluorescence allows the generation of informative 3-d plots of particle size vs. fluorescence vs. count frequency. This system originally used $325 \mathrm{~nm}$ radiation from a helium-cadmium laser but again has since been modified for use with shorter UV radiation so as to achieve the excitation of additional biological fluorophores.

Seaver et al ${ }^{6}$ has recently developed an instrument which similarly records simultaneously an estimate of particle size (in this case based on elastic scatter) together with intrinsic particle fluorescence. Again, the ability to produce plots of fluorescent intensity against scattered light signal has illustrated the potential of such dual-parameter measurements in the achievement of particle type discrimination.

Any monitoring system must aim for a minimum level of 'false positives'. In the case of biological aerosols in a military scenario, such events can lead to considerable effort being expended in taking protective measures and if too frequent, will result in the monitor being mistrusted or, at worst, disregarded completely. In an attempt to further reduce the occurrence of false positives, such as may occur when non-biological particles are present with similar size and fluorescence signature to biological particles, additional characteristics must be determined from the scattering particle. One method of achieving this is to examine the spatial pattern of light scattered elastically from the particle, from which both particle size and shape information may be deduced.

\section{SPATIAL LIGHT SCATTERING}

The manner in which a particle spatially scatters incident light is a complex function of the size, shape, structure, and orientation of the particle, as well as of the properties of the illuminating radiation (wave-length, polarization state). With suitable control of some of these variables it is possible to determine parameters relating to the shape and structure of the scatterer. This spatial light 
scattering analysis, also in certain geometries referred to as Two-dimensional Anglular Optical Scattering (TAOS) ${ }^{7}$, has therefore been attracting considerable attention for particle characterisation. Dick $e t a l^{8}$ (1998) have used multi-angle azimuthal measurements to determine the spherical and nonspherical fractions of laboratory and atmospheric aerosols; Holler et al (1998) used TAOS to examine clusters of Bacillus subtilis spores and found that 'island' structures were observable in the spatial scattering patterns and that the number of these islands increased per unit solid angle with increase in cluster diameter; Sachweh et $a l^{9}$ (1999) investigated spatial light scattering using three detection geometries and found not only good particle shape characterisation was obtainable but also that, for supermicron particles, the surface structure of the particle contributed to the scattering pattern provided the size of surface features substantially exceeded the wavelength of illumination.

The authors too have explored the potential of spatial light scattering analysis ${ }^{10-12}$ for particle shape characterisation and this work has resulted in a number of real-time monitoring systems for application in, for example, environmental aerosol monitoring ${ }^{13}$ and asbestos fiber detection ${ }^{14}$. The prototype instrument described in this paper seeks to combine the advantages which spatial light scattering analysis offers in terms of particle shape classification with those, demonstrated by the workers referred to earlier, offered by intrinsic fluorescence.

\section{INSTRUMENTATION}

The prototype multiparameter scattering instrument is shown schematically in Figure 1. The instrument employs a continuous-wave 266nm laser (Coherent Verdi Nd:Vanadate 532nm with external doubler to 266nm; Coherent Inc., Santa Clara, CA, USA). This laser, delivering up to $200 \mathrm{~mW}$ of output power in the UV, is used to produce both the spatial elastic-scatter data from the particle (from which size and shape parameters are derived) and the necessary particle fluorescence excitation. 
In operation, particle laden air from a test aerosol is drawn through a scattering chamber at a rate of approximately 1 litre per minute. A filtered flow of sheath air aerodynamically focuses the sample airstream and constrains it to pass through the circularly polarized $266 \mathrm{~nm}$ beam from the UV laser. The airflow also has the effect of preferentially aligning elongated particles with their long axis parallel to the flow. This is an important attribute in classifying particle shape from the variation in azimuthal scattering ${ }^{13,14}$. At the point of intersection with the sample airflow, the beam dimensions are $\sim 2 \mathrm{~mm}$ width by $\sim 100 \mu \mathrm{m}$ depth, ensuring that for particle number concentrations of up to $\sim 10^{5} / 1$, particle coincidences in the beam are rare. Light scattered both elastically and inelastically by the particle between angles of $30^{\circ}$ to $140^{\circ}$ to the beam axis is reflected from an ellipsoidal mirror and onto a photomultiplier detector via a long-pass optical filter (295nm, WG-295, CVI Laser Corp., Albuquerque, NM, USA). The filter thus allows only the particle fluorescence signal to reach the detector.

Light scattered by the particle in the forward direction (between angles of $4^{\circ}$ and $30^{\circ}$ ) is imaged onto a multi-pixel high gain hybrid photodiode detector, HPD (Delft Electronic Products BV, the Netherlands). It is this spatial scattering information which provides the particle shape classification. HPDs are similar to first generation image intensifiers, employing a photocathode to convert optical to electron flux, and accelerating the electrons (typically through $\sim 10 \mathrm{kV}$ ) into a multi-pixel silicon diode structure where multiple electron-hole pairs are produced. Gains of up to 2,000 are achievable within a compact, robust, and very low-power package. The HPD device used in our instrument has a custom designed arrangement of 31 pixels in a $15 \mathrm{~mm}$ diameter ring-wedge arrangement as shown in Figure 2. It comprises three concentric rings together with a center aluminized beam stop (shaded). The innermost ring, labelled 2 , is continuous and provides the opportunity for particle detection regardless of particle orientation. The next two rings are divided into 6 and 24 segments respectively to allow azimuthal and radial measurements of scattered light intensity.

The data acquisition electronics are situated close to the HPD device to minimize electrical noise interference. The electronics comprise 32 processing channels, 31 of which process data from the 
HPD whilst the $32^{\text {nd }}$ processes data from the fluorescence PMT detector at the opposite end of the chamber. Each channel comprises a transimpedance amplifier, dc-restoration circuitry (which removes any dc levels from the signals caused by background chamber illumination etc) followed by signal integration and digitization. The inner ring of the HPD acts also as a particle-detect trigger, its output being passed to a comparator to initiate the particle detect sequence. Once the output level of this channel is above a user defined threshold the control logic forces the integrators for all 32 channels to commence integration. Integration terminates once the signal level on the trigger channel falls below the threshold. The integration period is typically $2 \mu \mathrm{s}$, corresponding to the time of flight of a particle through the beam. The integrators hold their respective signal levels until they are passed to one of four 12-bit analogue-to-digital converters (ADC), one for every eight channels. The subsequent digitized scattering and fluorescence values are multiplexed via serial data lines to the host computer for buffering and storage. Data may be acquired for particle throughput rates of up to $\sim 5,000$ particles/s.

\section{Data Display}

Although spatial scattering and fluorescence data are stored in real-time for subsequent analysis, the prototype instrument allows a simple real-time graphical display of the incoming data for diagnostic purposes. Figure 3 show the screen display for a fiber particle (gypsum). The example also illustrates the effect which the inlet nozzle has on preferentially aligning elongated particles with their long axis parallel to the airflow. In the case of fiber particles, this results in essentially horizontal scattering (refer to Figure 1), giving peak outputs at detector pixels 12 and 24 on the outer pixel ring, as shown in Figure 3. 'Tr' is the output of the trigger detect pixel, No. 2; ' $\mathrm{Fl}$ ' is the output of the fluorescence photomultiplier tube detector.

\section{Detector Gain Correction and Calibration}

Before further processing, the data must be corrected for channel gain variations which arise in part from photocathode efficiency variation across the input window of the HPD device. This is achieved by collecting data from known spherical particles such as polystyrene latex and normalizing the 
channel responses to these particles (on the grounds that known spherical particles should ideally produce equal response in all detectors within a specified annulus). The data presented in this paper have been corrected in this way.

\section{PARTICLE CLASSIFICATION}

A convenient method of processing spatial light scatter data to yield particle shape classification indices is through the use of Scatter Intensity versus Asymmetry Factor plots ${ }^{13}$. The scatter intensity here is taken as the mean around the whole $360^{\circ}$ of an azimuthal ring of detectors and this gives a non-linear function related to the scattering cross-section of the particle which may itself be correlated with spherical equivalent particle diameter via Mie theory ${ }^{15}$. The Asymmetry Factor, Af, is a measure of the variation of the scattered light intensity around the azimuthal ring. It may be calculated using data from either the 6-pixel middle ring or the 24-pixel outer ring on the DEP HPD. The expression for calculation of Af is:

$$
A f=\sqrt[k]{\sum_{i=1}^{n} \bar{E}-E_{i}^{2}} /
$$

where $\mathrm{n}$ is the number of pixels in the ring, $\mathrm{E}$ is the output value of each pixel, $\mathrm{E}-$ bar the mean of all $\mathrm{E}$ values, and $\mathrm{k}$ is a constant to render the maximum possible value of Af to be 100 . This maximum Af will occur when all but one pixels in an azimuthal ring are zero. By applying this condition to equation $1, \mathrm{k}$ can be shown to be

$$
k=100 / \longdiv { n ( n - 1 ) }
$$

Clearly, in an ideal situation (with no experimental uncertainties), spherical particles which generated equal signals at each detector in the azimuthal ring will produce an $\mathrm{Af}=0$. 


\section{Scattering Asymmetry Performance Limits}

As described above, the instrument uses the azimuthal variation of scattered light intensity to determine a particle shape (or asphericity) index Af. It was necessary first, therefore, to determine the instrumental limits of minimum detectable azimuthal asymmetry and the linearity of the detector pixel responses throughout their useable dynamic range. The detection limits are set by the signal-to-noise ratio in the detector and by bandwidth and $\mathrm{ADC}$ conversion limitations within the signal acquisition and processing electronics. In order to determine this information, a pulsed light emitting diode $(\sim 540 \mathrm{~nm})$ was used to simulate particle transits through the laser beam by uniformly illuminating the whole of the HPD detector. The output of the LED was adjusted from zero to the maximum signal which could be accommodated by the processing electronics and this simulated 'particle' data recorded and processed to yield a Scatter Intensity versus Af plot as described above.

Figure 4a shows the Scatter Intensity versus Af plot for the simulated particle data. As would be expected for uniform illumination of azimuthal pixels (simulating scattering by a perfect sphere ${ }^{15}$ ), the calculated Af values are close to zero throughout the majority of the LED output intensity range. However, at low intensities the calculated Af values increase markedly. This is more apparent in Figure $4 \mathrm{~b}$ which shows the same data plotted on logarithmic ordinate and abscissa. Here the value of calculated Af increases almost linearly with decreasing input signal magnitude. This is principally attributable to shot noise in the detected analog signals from the detector pixels causing significant variation in the digitised signal values which, in turn, result in increased Af values. The solid line marked on Figure $4 \mathrm{~b}$ indicates the approximate limit of performance of the system in terms of particle shape characterisation: perfectly spherical scatterers would produce data points along this line; Af values to the right of the line may be interpreted as azimuthal variations in scattering arising predominantly from non-spherical particle shape or structure. 


\section{PRELIMINARY EXPERIMENTAL RESULTS}

Since the HPD was of a new and untried design, the first objective was to evaluate the spatial scattering data it recorded and assess the potential of these data for particle classification. For ease of optical alignment, this initial assessment of the HPD was carried out with an illumination wavelength of $532 \mathrm{~nm}$ rather than $266 \mathrm{~nm}$. This also allowed unwanted sources of background scattering within the scattering chamber to be identified and minimised before the implementation of the shorter wavelength. The use of $532 \mathrm{~nm}$ illumination precluded the measurement of particle fluorescence at this stage.

Figure 5 shows Scatter Intensity versus Af plots for a variety of aerosol types: water droplets, hematite grains, BG spores (Bacillus subtilis var. niger), and gypsum dust. The hematite is prolate ellipsoidal in shape with major axis $\sim 2-3 \mu \mathrm{m}$ and aspect ratio typically $3: 1$. It is similar in size and shape to the BG spores. The gypsum has an irregular fibrous morphology, with aspect ratios from $\sim 3: 1$ to $8: 1$ and lengths up to $\sim 10 \mu \mathrm{m}$. Each aerosol was generated independently. The dry powder hematite and gypsum were aerosolized using a compressed air jet into a ballast chamber from which the instrument sample flow was drawn. The BG spores were aerosolised into the ballast chamber using a nebuliser. The water droplets were sprayed directly over the vicinity of the instrument inlet. (The spray was independently measured using an Aerodynamic Particle Sizer (APS model 3320, TSI Inc., St. Paul, MN, USA) which indicated the droplets were polydisperse with a distribution of aerodynamic diameters from $\sim 1 \mu \mathrm{m}$ to greater than $10 \mu \mathrm{m}$, although the largest of these droplets lay outside the measurable size range of our prototype instrument.

Features to note from Figure 5 are:

(a) As would be expected, scattering data for the droplets lie close to the instrument performance limit for spherical particles with Af values generally less than unity. The droplets were clearly distinguishable from the non-spherical hematite and gypsum particles which exhibit Af values from $\sim 5$ to $\sim 60$. 
(b) The results suggest the aerosolization process did not completely break down the dry hematite into the fundamental particles. Agglomerates up to the top of the measurable range $(\sim 10 \mu \mathrm{m})$ were recorded. The lower Af values of these agglomerates indicates they assumed a more spherical shape than the individual ellipsoidal grains.

(c) There is significant overlap between the hematite, gypsum (at their lower size range), and BG spore data. This might be expected as the morphologies of the hematite and lower aspect ratio gypsum particles are somewhat similar to that of the spores. It is this type of particle classification ambiguity which the additional parameter of particle fluorescence is intended to mitigate.

\section{Spatial Scattering and Fluorescence Measurements}

Preliminary data have been acquired using the $266 \mathrm{~nm}$ radiation for simultaneous spatial scattering and fluorescence excitation. The spatial scattering data obtained at this wavelength was used to produce the Scatter Intensity vs Af plot shown in Figure 6. Data are presented for $1 \mu \mathrm{m}$ and $3 \mu \mathrm{m}$ diameter polystyrene latex PSL spheres (Dow Corning), 1.7 $\mu \mathrm{m}$ diameter fluorescent latex spheres (Polyscience Inc. Fluoresbright), gypsum, and BG spores. Unfortunately, hematite samples were not available at the time of these tests.

Figure 6 shows all three varieties of PSL spheres close to the instrument performance limit for spherical particles although the range of Asymmetry Factor, Af, values was comparatively large for the $1 \mu \mathrm{m}$ PSL. It is suspected that this was as a result of significant occurrences of doublets and higher order multiplets being present in the aerosol from the nebuliser. The BG spore data and gypsum data once again show a substantial overlap in the 10-30 Af range, making differentiation of these particles impossible from the scatter intensity and Af data alone. The spread of scatter intensity levels for all three varieties of PSL is larger than would be expected given the inherent size uniformity of these materials. The principal reason for the spread is the variation in beam intensity across the scattering volume (ie: the intersection between the beam and the sample airflow). Whilst the airflow column 
diameter is less than the beam width, the Gaussian profile of the beam makes completely uniform illumination of the scattering volume impossible.

Figure 7 shows the same particle data as Figure 6 but in this case the degree of intrinsic particle fluorescence has been introduced in addition to scatter intensity and Af. The graph shows the ratio of particle fluorescence divided by scatter intensity as a function of particle Asymmetry Factor. This is a simplistic approach to normalising the fluorescence signal for particle volume but nevertheless it provides an effective means of data separation. As can be seen from Figure 7, the fluorescent $1.7 \mu \mathrm{m}$ PSL is, as would be expected, clearly differentiated from the non-fluorescent PSL varieties, having an order of magnitude greater fluorescence/scatter intensity ratio. The outlying data points for the fluorescent PSL (up to values of $\mathrm{Af}=10$ ) occur at virtually constant Fluorescence/Scatter Intensity ratios, again suggesting these are the product of doublets and multiplets in the aerosol.

More importantly, the BG spores and Gypsum, indistinguishable on the Scatter intensity versus Af plot, are here widely separated as a result of the substantial differences in intrinsic fluorescence of the two materials.

\section{DISCUSSION}

The instrument described in this paper is at an early stage of development but has nevertheless yielded encouraging results so far. The unique combination of particle shape/size characterization from spatial scattering analysis together with intrinsic fluorescence measurement offers a powerful method of classifying airborne particle types and, in particular, offers potentially enhanced discrimination between biological and non-biological particles. We are currently in the process of refining the instrument to improve the uniformity of scattering volume irradiance and further reduce background optical noise. This will be followed by an extensive programme of experimental trials covering a wide variety of biological and non-biological materials to fully assess the degree of particle discrimination which may be achieved in the field. Beyond this, there exists the possibility of spectrally interrogating 
the fluorescence signatures of the particles under test. Other workers ${ }^{3,4}$ have shown that such spectra can provide additional characteristic information to enhance discrimination.

The use of a single continuous wave ultra-violet laser to acquire both elastic scatter and fluorescence data offers some advantages over systems ${ }^{3-6}$ which utilize a pulsed UV source triggered using a separate continuous-wave visible laser: firstly it simplifies the opto-mechanical arrangement of the light scattering chamber and removes the need for the high speed triggering circuitry which is required in the pulsed UV laser systems; secondly, the short UV wavelength (266nm compared to 532-650nm used in our previous shape classification instruments ${ }^{13,14}$ ) theoretically improves the sensitivity of the instrument to particle shape variations, especially for the smaller micron-sized range into which many airborne bio-organisms fall; thirdly, since the particle is exposed to UV power levels which are far lower than experienced in the pulsed laser fluorescence systems $(200 \mathrm{~mW}$ for $\sim 2 \mu \mathrm{s}$ compared with tens of Watts for nanoseconds), the potential problems of photo-bleaching of the fluorescent molecules within the particle which can occur at high irradiances are lessened. However against these advantages, the continuous-wave laser system we are using is large and delicate, and impractical for use in a field instrument. Such field implementation will require the development of more compact and robust continuous-wave UV laser sources.

\section{ACKNOWLEDGEMENTS}

This work has been supported by grants from the UK Defence Evaluation Research Agency, Porton Down. 


\section{REFERENCES}

1. R. G. Pinnick, S. C. Hill, P. Nachman, J. D. Pendleton, G. L. Fernandez, M. W. Mayo, and J. G. Bruno, "Fluorescence particle counter for detecting airborne bacteria and other biological particles", Aerosol Science and Technology 23, 653-664 (1995).

2. P. Nachman, G. Chen, R. G. Pinnick, S. C. Hill, R. K. Chang, M. W. Mayo and G. L. Fernandez, "Condition sampling spectrograph detection system for fluorescent measurements of individual airborne biological particles", Appl. Opt. 35, 7, 1069-1076 (1996).

3. G. Chen, P. Nachman, R. G. Pinnick, S. C. Hill, and R. K. Chang, " Conditional firing aerosol fluorescence spectrum analyser for individual airborne particles with pulsed 266nm laser excitation", Optics Letters 21, 16, 1307-1309 (1996).

4. Yongle Pan, S. Holler, R. K. Chang, S. C. Hill R. G. Pinnick, S. Niles, and J. R. Bottiger, "Single-shot fluorescence spectra of individual micrometer-sized bioaerosols illuminated by a 351- or 266nm ultraviolet laser”, Optics Letters 24, 2, 116-118 (1999).

5. P. P. Hairston, J. Ho, and F. R. Quant, "Design of an instrument for real-time detection of bioaerosols using simultaneous measurement of particle aerodynamic size and intrinsic fluorescence”, J. Aerosol Sci. 28, 3, 471-482 (1997).

6. M. Seaver, J. D. Eversole, J. J. Hardgrove, W. K. Cary Jr, and D. C. Roselle, "Size and fluorescence measurements for field detection of biological aerosols", Aerosol Science and Technology 30: (2) 174-185 (1999).

7. S. Holler, Yongle Pan, R. K. Chang and J. R. Bottiger, "Two-dimensional angular optical scattering for the characterisation of airborne microparticles", Optics Letters 23, 18, 14891491 (1998).

8. W. D. Dick, P. J. Ziemann, Po-Fu Huang, and P. H. McMurray, "Optical shape fraction measurements of submicrometre laboratory and atmospheric aerosols", Meas. Sci. and Tech. 9, 183-196 (1998). 
9. B. Sachweh, H. Barthel, R. Polke, H. Umhauer, and H. Buttner, "Particle shape and structure analysis from the spatial intensity pattern of scattered light using different measuring devices", J. Aerosol Sci. 30, 10, 1257-1270 (1999)

10. E. Hirst, P. H. Kaye, and J. R. Guppy, "Light Scattering from Non-spherical Airborne Particles: Experimental and Theoretical Comparisons”. Appl. Opt. 33, 30, 7180-7186 (1994).

11. E. Hirst and P. H. Kaye, "Experimental and Theoretical Light Scattering Profiles from Spherical and Non-spherical Particles”. J. Geophys. Res. (Atmospheres) 101, D14, 19,23119,235 (1996).

12. P. H. Kaye, "Spatial Light Scattering as a Means of Characterising and Classifying Nonspherical Particles”. Meas. Sci. and Tech. 9, 2, 141-149 (1998).

13. P. H. Kaye, K. Alexander-Buckley, E. Hirst, and S. Saunders, "A Real-time Monitoring System for Airborne Particle Shape and Size Analysis”. J. Geophys. Res. (Atmospheres) 101, D14, 19,215-19,221 (1996).

14. E. Hirst, P. H. Kaye and Z. Wang-Thomas, "A Neural Network based Spatial Light Scattering Instrument for Hazardous Airborne Fiber Detection”. Appl. Opt. 36, 24, 6149$6156(1997)$.

15. C.F. Bohren and D. R. Huffman, Absorption and Scattering of Light by Small Particles. (Wiley, New York, 1983). 


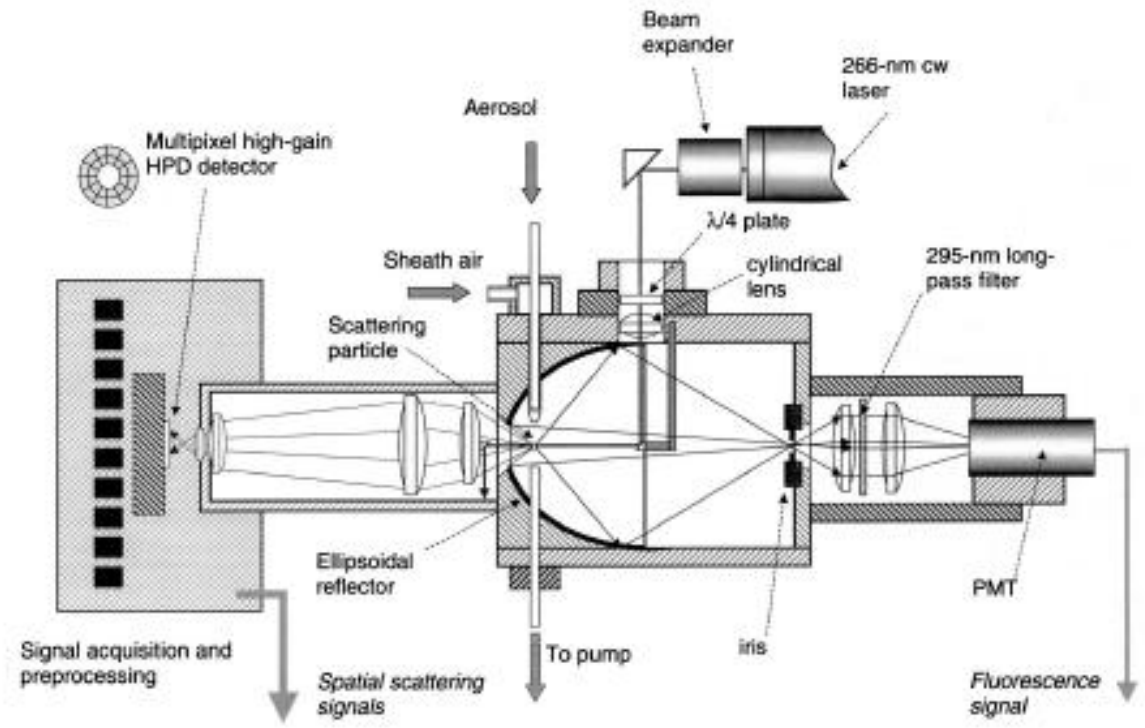

Fig 1. Schemativ diagram of the scattering chamber for the multiparameter scattering fluorescence instrument. The overall length of the chamber is $35 \mathrm{~cm}$.

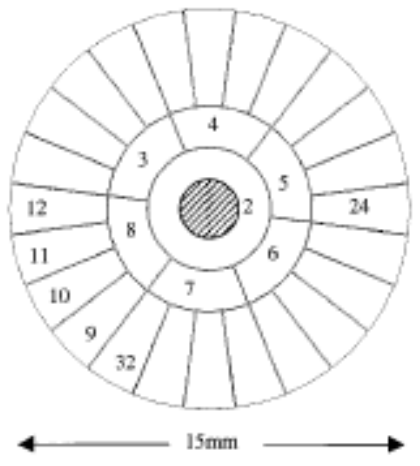

Fig. 2. Layout of the 31-pixel detector geometry used in the HPD epatiol soattering detector. At the center is an duminized beom stop. The output of the innermost annulus ( 2 ) is used as a particle detect trigger, and the segmented outer ringe proride azimuthal scattering data from which an coseosment of particle shape is made.

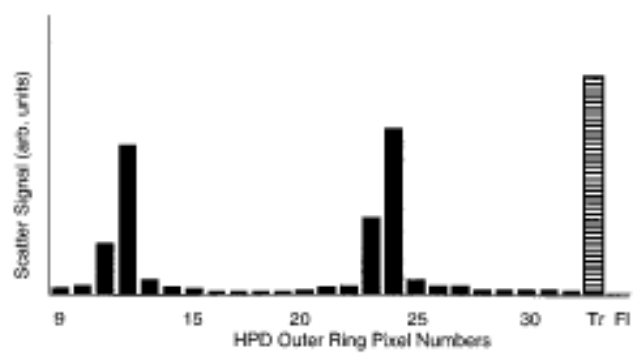

Fig. 3. Example dioplay screen showing the output of the 24 outer-ring detector pixels resulting from a vingle gypoum fiber passing through the ocattering volume. The vertical orientation of the fiber produces horizontal soattering as indicated by the high values in pixels 12 and $24 . \mathrm{Tr}$, output of the trigger detect pixel 2; Fl, output of the fluorescence PMT detector 

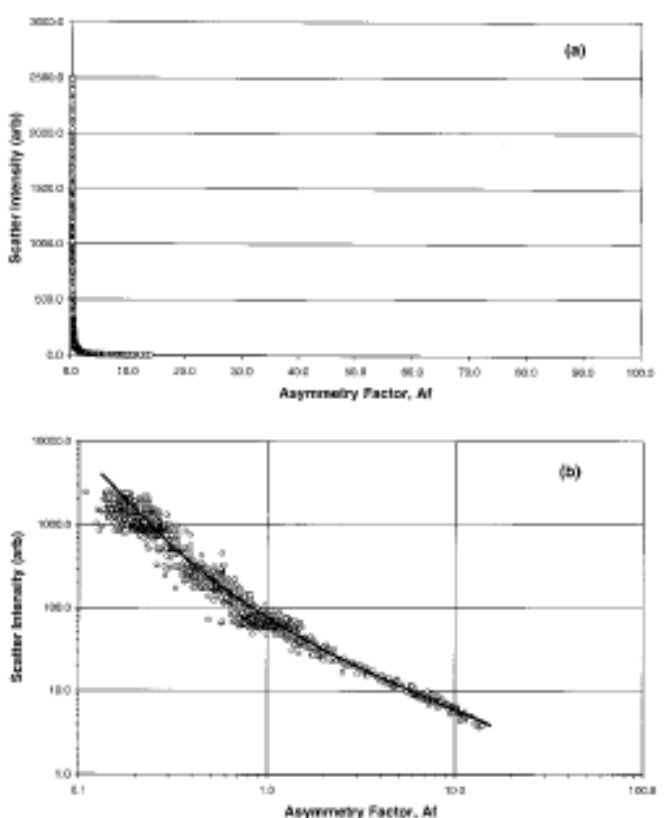

Fig 4. (a) Sontter intensity vervus Af plot for aimulated ophericol particle data (produced by uniform illumination of the HPD from a puleed LED source). Ideally wuch data should lie entirely along the ordinate $(\mathrm{Af}=0)$ correoponding to perfectly opherical ocatterera. (b) As in (a) but with logorithmic scalea. The solid curve represents the noise-limited performance of the system in terms of particle ahape characterization: Perfectly spherical acatterere would produce data points along this curve, and nonepherical scatterere mould result in data points to the right of the curve. 


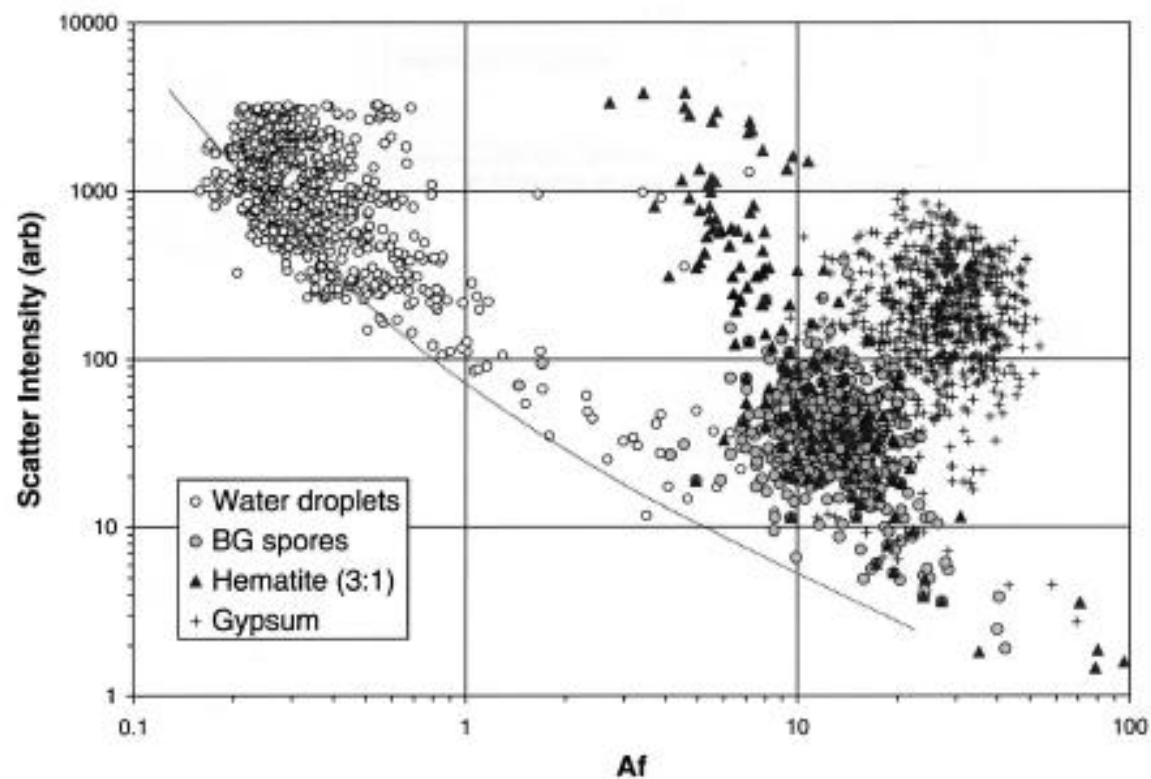

Fig. 5. Scatter intensity vereus Af plote recorded at a 5\$2-nm wavelength for aeroools of water dropleto, hematite grains, BG opores, and gypeum dust. The dotted curve again represents the limit of performance for perfectly opherical ecatterers.

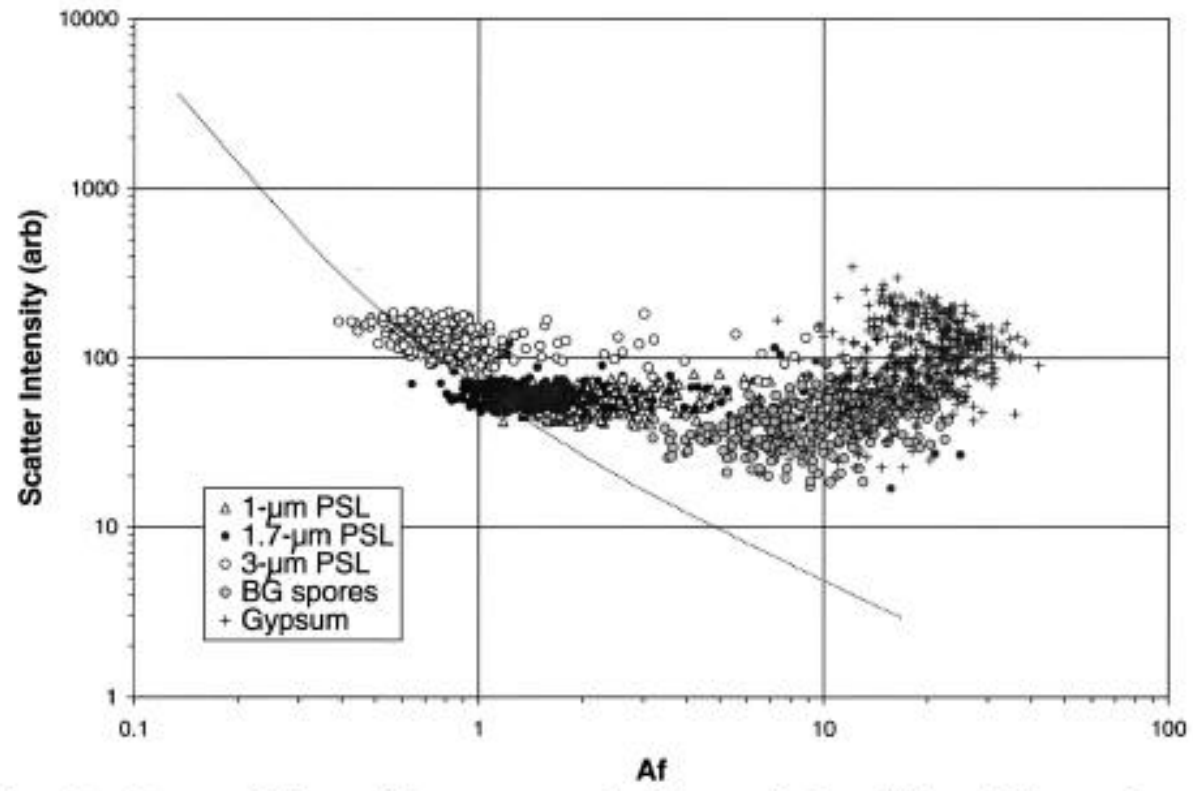

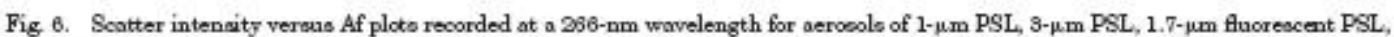
BG opores, and gypoum dust. The dotted curve again repreoents the limit of performanoe for perfectly opherical ocatterers. 


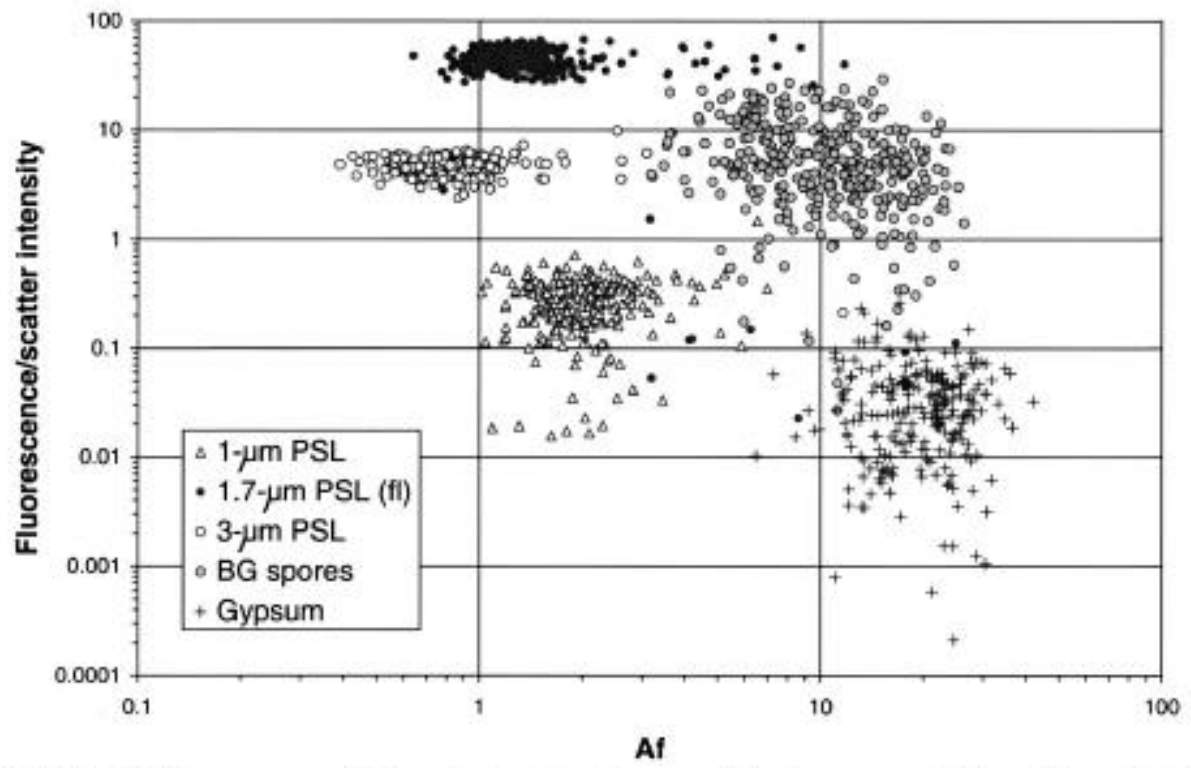

Fig. 7. Graph of fluorescence amplitude and ocatter intensity versus Af for the same aerool data as chown in Fig. 6. 